1 Departamento de Análises Clínicas

e Toxicológicas, Faculdade de Farmácia, Universidade Federal de São João Del Rei (UFSJ), Campus Centro Oeste Dona Lindu, Divinópolis, MG, Brasil 2 Departamento de Clínica

Médica, Faculdade de Medicina, Universidade Federal de Minas Gerais (UFMG), Belo Horizonte, MG, Brasil

${ }^{3}$ Departamento de Pediatria,

Faculdade de Medicina, UFMG, Belo Horizonte, MG, Brasil

${ }^{4}$ Departamento de Análises Clínicas e Toxicológicas,

Faculdade de Farmácia, UFMG, Belo Horizonte, MG, Brasil

${ }^{5}$ Departamento de Endocrinologia

e Metabolismo, Instituto de

Educação e Pesquisa da Santa

Casa de Belo Horizonte, Belo

Horizonte, MG, Brasil

Correspondence to: Caroline Pereira Domingueti Departamento de Análises Clínicas e Toxicológicas,

Faculdade de Farmácia

Universidade Federal de

São João Del Rei

Rua Sebastião Gonçalves

Coelho, 400, Chanadour

35501-296 - Divinópolis, MG, Brasil

caroldomingueti@yahoo.com.br

Received on Nov/30/2015

Accepted on Dec/8/2015

DOI: 10.1590/2359-3997000000151

\section{Evaluation of creatinine-based and cystatin C-based equations for estimation of glomerular filtration rate in type 1 diabetic patients}

\author{
Caroline Pereira Domingueti', Rodrigo Bastos Fóscolo², Ana Cristina \\ Simões e Silva ${ }^{3}$, Luci Maria S. Dusse ${ }^{4}$, Janice Sepúlveda Reis ${ }^{5}$, Maria das \\ Graças Carvalho ${ }^{4}$, Ana Paula Fernandes ${ }^{4}$, Karina Braga Gomes ${ }^{4}$
}

\begin{abstract}
Objective: Several formulas based in different biomarkers may be used to estimate glomerular filtration rate (GRF). However, all of them have some limitations, and it is very important to evaluate their performances in different groups of patients. Therefore, we compared GFR, as estimated by creatinine-based and cystatin C-based equations, according to albuminuria, in type 1 diabetes (T1DM), in an observational case-control study. Subjects and methods: T1DM patients were classified according to albuminuria: normoalbuminuric $(n=63)$, microalbuminuric $(n=30)$, macroalbuminuric $(n=32)$. GFR was calculated using creatinine-based and cystatin C-based (aMDRD, CKD-EPIcr, CKD-EPIcys, Maclsaac, Tan and CKD-EPIcrcys) equations. Spearman Correlation was used to evaluate the correlation of GFR estimated by the formulas with albuminuria. ROC curves were constructed to compare AUCs of GFR estimated by equations, in reference to macroalbuminuria. Sensibility, specificity and accuracy were calculated for a cut-off $<60 \mathrm{~mL} / \mathrm{min} / 1.73 \mathrm{~m}^{2}$. Results: GFR estimated by creatinine-based and cystatin $\mathrm{C}$-based equations significantly differed among normoalbuminuric, microalbuminuric and macroalbuminuric patients. Spearman correlation and AUCs of GFR estimated by creatinine-based and cystatin C-based formulas were very similar to each other, though cystatin C-based equations presented better correlation with albuminuria and higher AUCs than the creatinine-based ones, and the best accuracy to detect macroalbuminuric patients. Conclusion: Although GFR estimated by all creatinine-based and cystatin C-based equations permitted the differentiation between T1DM patients, according to albuminuria, cystatin C-based equations presented best accuracy to detect macroalbuminuria in T1DM patients and should be considered in the clinical routine in order to increase the possibility of early diagnostic of chronic renal disease. Arch Endocrinol Metab. 2016;60(2):108-16
\end{abstract}

Keywords

Albuminuria; cystatin C; estimated glomerular filtration rate; diabetic nephropathies; diabetes mellitus, type 1

\section{INTRODUCTION}

$\mathrm{D}$ iabetic nephropathy is characterized by a progressive increase in urinary albumin excretion (UAE). Moreover, it is associated to glomerular filtration decline and, eventually, renal failure (1). It is the most important cause of end stage renal disease (ESRD), and an independent risk factor for cardiovascular disease (2). It is estimated that 25 to $40 \%$ of type 1 (TlDM) and type 2 (DM2) diabetic patients develop renal disease 20 to 25 years after the diagnosis of diabetes (3).

Diabetic nephropathy can be divided into stages, according to UAE. The initial stage or incipient nephropathy is characterized by microalbuminuria, while the advanced stage or clinical nephropathy is characterized by macroalbuminuria (4). UAE is the most important biomarker of renal parenchyma injury, and is used to diagnose and to establish the prognosis of nephropathy (5). The evaluation of UAE must be conducted along with glomerular filtration rate (GFR) estimation to assess renal function in diabetic patients, since some patients with normoalbuminuria present a decline in $\operatorname{GFR}(4,6)$.

The most used creatinine-based equations to estimate GFR in clinical practice are Cockcroft-Gault (CG) and abbreviated Modification of Diet in Renal Disease $(\mathrm{aMDRD})(7,8)$. However, the CG formula tends to overestimate GFR, especially in patients with severe renal disease, and aMDRD tends to subestimate GFR of persons with normal renal function (9-11). Besides, 
the CG formula was developed before standardization of creatinine assays and cannot be re-expressed for use with these assays (12). The Chronic Kidney Disease Epidemiology Collaboration (CKD-EPI) research group developed a new creatinine-based equation to estimate GFR as an attempt to overcome the limitations of the other formulas (13). However, this new equation also has some limitations, since it has not been extensively studied in different populations yet, and there is still no ideal equation to estimate GFR $(14,15)$.

Cystatin $\mathrm{C}$, a new endogenous biomarker of renal function, was also proposed to assess GFR (16-18). It is a cysteine protease inhibitor with low molecular-weight $(13.3 \mathrm{kDa})$ that is freely filtered and almost taken up and degraded but not secreted by proximal tubular cells. Cystatin $\mathrm{C}$ is one of the biomarkers that meet the conditions necessary for an endogenous substance to be marker of the GFR. It is produced by the nucleated cells in a constant amount, released into bloodstream with a halp-life of 2 hours, and its serum concentration does not depend on muscle mass and protein intake, which reduces the influence of age, gender, weight (19-21). Some authors have suggested that cystatin C-based equations are equal or superior to creatininebased ones and to creatinine clearance, especially to detect early stages of chronic kidney disease $(22,23)$. However, they emphasize that further studies are necessary to evaluate its efficacy in different groups of patients and that cystatin $\mathrm{C}$ serum levels should be interpreted carefully because there are some factors that can affect its levels regardless of the renal function, such as thyroid dysfunction, glucocorticoids use and malignancies $(16,17,20)$.

In this study, we compared the estimation of GFR by different creatinine-based and cystatin C-based equations in TIDM patients classified according to levels of albuminuria.

\section{SUBJECTS AND METHODS}

The clinical records of all consecutive 140 TIDM patients receiving assistance at the Endocrinology Ambulatories of University Hospital (Hospital das Clínicas) and Santa Casa/Belo Horizonte, Brazil, from November 2011 to September 2012, for quarterly or biannual check up, were analyzed and 15 were excluded from the study due to presence of at least one of the following exclusion criteria: hepatic disease, alcoholism, haemostatic abnormalities, malignant diseases, acute infectious, history of kidney transplantation, pregnancy or undergoing hemodialysis. After application of exclusion criteria, 125 patients with clinical and laboratorial diagnosis of TIDM (24), 18 to 60 years old, were selected for this study.

All procedures performed in this study were in accordance with the 2000 Declaration of Helsinki as well as the Declaration of Istanbul 2008. The Research Ethics Committee of Federal University of Minas Gerais approved this study (CAAE - 0392.0.203.000-11). An informed consent was obtained from all individual participants included in the study. The research protocol did not interfere with any medical recommendations or prescriptions.

Data regarding age, sex, weight, height, time of diagnosis of diabetes, presence of retinopathy and neuropathy, use of antihypertensive, statin and acetylsalicylic acid (AAS) were obtained from medical records. Fasting glucose, creatinine and urea were determined by enzymatic method; albumin was assessed by colorimetric method and $\mathrm{HbAlc}$ was determined by immunoturbidimetric method; using Johnson \& Johnson dry chemistry technology kits (Ortho Clinical Diagnostics $^{\circledR}$ ) and VITROS 4600 analyzer. ELISA (Human Cistatin C kit - Biovendor ${ }^{\circledR}$ ) was used to measure Cystatin C. UAE was determined in urine samples collected after at least 4 hours of urinary retention, and urinary albumin was normalized by urinary creatinine. Urinary albumin was evaluated by immunoturbidimetric method and urinary creatinine was assessed by enzymatic method, using Johnson \& Johnson dry chemistry technology kits (Ortho Clinical Diagnostics ${ }^{\circledR}$ ) and VITROS 4600 analyzer.

Normoalbuminuria was defined as $<30 \mathrm{mg}$ of albumin/g of creatinine, microalbuminuria as $\geq 30$ and $<300 \mathrm{mg}$ of albumin/g of creatinine and macroalbuminuria as $\geq 300 \mathrm{mg}$ of albumin/g of creatinine. The presence of microalbuminuria or macroalbuminuria was confirmed in two out of three occasions, over a period between three and six months (6).

The estimated GFR was calculated using two creatinine-based equations: eGFR-aMDRD expressed for standardized serum creatinine (25) and eGFR-CKDEPIcr (13); three cystatin C-based equations: eGFRCKD-EPIcys (16), eGFR-MacIsaac (17), eGFR-Tan (18); and one equation based on creatinine and cystatin C: CKD-EPIcrcys (16) (Table 1). CG formula was not used because it cannot be re-expressed for standardized serum creatinine (12). 
Table 1. Creatinine-based and cystatin C-based equations used to estimate GFR

\begin{tabular}{|c|}
\hline $\begin{array}{l}\text { aMDRD } \\
\text { eGFR }\left(\mathrm{mL} / \mathrm{min} / 1.73 \mathrm{~m}^{2}\right)=175 \times \text { serum creatinine }(\mathrm{mg} / \mathrm{dL})^{-1.154} \times \text { age } \\
\text { (years) })^{-0.203} \times 0.742 \text { (if female) } \times 1.212 \text { (if black) }\end{array}$ \\
\hline $\begin{array}{l}\text { CKD-EPIcr } \\
\text { Male: }\end{array}$ \\
\hline $\begin{array}{l}\text { Serum creatinine } \leq 0.9 \mathrm{mg} / \mathrm{dL} \text { : eGFR }\left(\mathrm{mL} / \mathrm{min} / 1.73 \mathrm{~m}^{2}\right)=\alpha \times(\text { serum } \\
\text { creatinine in } \mathrm{mg} / \mathrm{dL} / 0.9)^{-0.411} \times(0,993)^{\text {age }}\end{array}$ \\
\hline $\begin{array}{l}\text { Serum creatinine }>0.9 \mathrm{mg} / \mathrm{dL} \text { : eGFR }\left(\mathrm{mL} / \mathrm{min} / 1.73 \mathrm{~m}^{2}\right)=\alpha \times \text { (serum } \\
\text { creatinine in } \mathrm{mg} / \mathrm{dL} / 0.9)^{-1.209} \times(0.993)^{\text {age }}\end{array}$ \\
\hline Female: \\
\hline $\begin{array}{l}\text { Serum creatinine } \leq 0.7 \mathrm{mg} / \mathrm{dL}: \mathrm{eGFR}\left(\mathrm{mL} / \mathrm{min} / 1.73 \mathrm{~m}^{2}\right)=\alpha \times(\text { serum } \\
\text { creatinine in } \mathrm{mg} / \mathrm{dL} / 0.7)^{-0.329} \times(0.993)^{\text {age }}\end{array}$ \\
\hline $\begin{array}{l}\text { Serum creatinine }>0.7 \mathrm{mg} / \mathrm{dL} \text { : eGFR }\left(\mathrm{mL} / \mathrm{min} / 1.73 \mathrm{~m}^{2}\right)=\alpha \times(\text { serum } \\
\text { creatinine in } \mathrm{mg} / \mathrm{dL} / 0.7)^{-1.209} \times(0.993)^{\text {age }}\end{array}$ \\
\hline $\begin{array}{l}\alpha=141 \text { for non-black male, } 144 \text { for non-black female, } 163 \text { for black male, } \\
166 \text { for black female }\end{array}$ \\
\hline CKD-EPIcys \\
\hline $\begin{array}{l}\left.127.7 \times \text { serum cystatin } C(\mathrm{mg} / \mathrm{L})^{-1.17} \times \text { age (years) }\right)^{-0.13} \times 0.91 \text { (if female) } \times 1.06 \\
\text { (if black) }\end{array}$ \\
\hline $\begin{array}{l}\text { Maclsaac } \\
\text { (84.6/serum cystatin } \mathrm{C} \text { in } \mathrm{mg} / \mathrm{L})-3.2\end{array}$ \\
\hline $\begin{array}{l}\text { Tan } \\
(87.1 / \text { serum cystatin } \mathrm{C} \text { in } \mathrm{mg} / \mathrm{L})-6.87\end{array}$ \\
\hline CKD-EPIcrcys \\
\hline $\begin{array}{l}177.6 \times \text { serum creatinine }(\mathrm{mg} / \mathrm{dL})^{-0.65} \times \text { serum cystatin } \mathrm{C}(\mathrm{mg} / \mathrm{L})^{-0.57} \times \text { age } \\
\text { (years) })^{-0.20} \times 0.82 \text { (if female) } \times 1.11 \text { (if black) }\end{array}$ \\
\hline
\end{tabular}

Patients were also classified according to eGFR assessed by creatinine-based and cystatin C-based equations into three groups: $\mathrm{eGFR} \geq 90 \mathrm{~mL} / \mathrm{min} / 1.73 \mathrm{~m}^{2}$; eGFR $\geq 60$ and $<90 \mathrm{~mL} / \mathrm{min} / 1.73 \mathrm{~m}^{2} ; \mathrm{eGFR}<60$ $\mathrm{mL} / \mathrm{min} / 1.73 \mathrm{~m}^{2}$.

Statistical comparisons were performed using SPSS software (version 20.0, SPSS). It was calculated that at least 27 subjects per group would be enough to detect differences of $20 \mathrm{~mL} / \mathrm{min} / 1.73 \mathrm{~m}^{2}$ in GFR between groups, with a standard deviation of 25 and test power of $90 \%$. The Shapiro-Wilk test was used to test the normality of the variables. Data normally distributed were expressed as mean \pm SD and were compared by ANOVA and $\mathrm{T}$ test. Data not normally distributed were expressed as median (percentiles 25\%-75\%) and were compared by Kruskal-Wallis $\mathrm{H}$ test and Mann-Whitney U test, followed by Bonferroni correction. Categorical variables were expressed as frequencies and compared using chi-square test $\left(\chi^{2}\right)$. Spearman Correlation was applied to evaluate the correlation of the GFR estimated by the formulas with UAE. eGFR was categorized into three groups $\left(\geq 90, \geq 60\right.$ and $<90$ and $\left.<60 \mathrm{~mL} / \mathrm{min} / 1.73 \mathrm{~m}^{2}\right)$ and was compared to normoalbuminuria, microalbuminuria and macroalbuminuria using chi-square test $\left(\chi^{2}\right)$ and kappa index was calculated. eGFR was also categorized into two groups $\left(\geq 60\right.$ and $\left.<60 \mathrm{~mL} / \mathrm{min} / 1.73 \mathrm{~m}^{2}\right)$ and was compared to normoalbuminuria or microalbuminuria and macroalbuminuria using chi-square test $\left(\chi^{2}\right)$ and odds ratio was calculated. ROC curves were constructed to compare the area under curve (AUC) of GFR estimated by different equations, in reference to macroalbuminuria. Sensitivity, specificity and accuracy were calculated for a cut-off $<60 \mathrm{~mL} / \mathrm{min} / 1.73 \mathrm{~m}^{2}$. Differences were considered significant when $\mathrm{p} \leq 0.05$.

\section{RESULTS}

Characteristics and clinical variables of the 25 TIDM patients included in this observational case-control study are presented in Table 2 .

Patients with macroalbuminuria presented lower BMI than patients with micro and normoalbuminuria ( $\mathrm{p}$ $=0.001$ and $\mathrm{p}=0.006$, respectively) and an increased frequency of retinopathy, more frequent use of antihypertensive, statin and AAS than patients with normoalbuminuria $(\mathrm{p}<0.001, \mathrm{p}<0.001, \mathrm{p}=0.001, \mathrm{p}=0.001$, respectively). There were no significant differences among the groups regarding to age, sex, time of diagnosis and frequency of neuropathy. Fasting glucose was lower in patients with microalbuminuria as compared to patients with normoalbuminuria $(\mathrm{p}<0.001)$. Higher levels of $\mathrm{HbAlc}$ and reduced serum albumin were observed in patients with macroalbuminuria, when compared to those with normoalbuminuria $(\mathrm{p}=0.009$ and $\mathrm{p}=0.007$, respectively). Patients with microalbuminuria presented increased levels of urea than patients with normoalbuminuria $(\mathrm{p}=0.001)$ and patients with macroalbuminuria had higher levels of urea, creatinine and cystatin $\mathrm{C}$ than the other groups $(\mathrm{p}<0.001)$. eGFR was reduced in patients with microalbuminuria compared to patients with normoalbuminuria, independent of the equation used to estimate GFR (eGFR-aMDRD, $\mathrm{p}=0.004$; eGFR-CKDEPIcr, $\mathrm{p}=0.001$; eGFR-Tan, $\mathrm{p}=0.003$; eGFR-MacIsaac, $\mathrm{p}=0.003$; eGFR-CKD-EPIcys, $\mathrm{p}=0.002$; eGFR-CKDEPIcrcys, $\mathrm{p}=0.006$ ). eGFR was also reduced in patients with macroalbuminuria as compared to patients with micro and normoalbuminuria, independent of the equation used to estimate GFR $(\mathrm{p}<0.001)$.

The classification of patients according to GFR as estimated by the formulas (eGFR $\geq 90 \mathrm{~mL} / \mathrm{min} / 1.73$ $\mathrm{m}^{2} ; \mathrm{eGFR} \geq 60$ and $<90 \mathrm{~mL} / \mathrm{min} / 1.73 \mathrm{~m}^{2}$; eGFR $<60$ $\mathrm{mL} / \mathrm{min} / 1.73 \mathrm{~m}^{2}$ ) was correlated to the classification of patients according to UAE (Table 3 ). The kappa in- 
Table 2. Clinical and epidemiological characteristics of diabetic patients classified according to albuminuria

\begin{tabular}{|c|c|c|c|c|}
\hline & $\begin{array}{c}\text { Patients with } \\
\text { normoalbuminuria }\end{array}$ & $\begin{array}{c}\text { Patients with } \\
\text { microalbuminuria }\end{array}$ & $\begin{array}{c}\text { Patients with } \\
\text { macroalbuminuria }\end{array}$ & $\mathbf{p}$ \\
\hline Number of Individuals (n) & 63 & 30 & 32 & \\
\hline Age (years) & $30 \pm 8$ & $37 \pm 11$ & $34 \pm 10$ & NA \\
\hline Sex/male (n, \%) & $25(55.6)$ & $6(13.3)$ & $14(31.1)$ & NA \\
\hline BMl $\left(\mathrm{kg} / \mathrm{m}^{2}\right)$ & $24.5 \pm 3.3$ & $26.1 \pm 3.4$ & $21.8 \pm 2.6^{\star \star x+}$ & $\begin{array}{c}0.001^{\star *} \\
0.006^{\dagger}\end{array}$ \\
\hline Time of diagnosis (years) & $18 \pm 8$ & $17 \pm 6$ & $20 \pm 5$ & NA \\
\hline Retinopathy (n, \%) & $18(32.7)$ & $14(25.5)$ & $23(41.8)^{\star \star}$ & $<0.001^{\star *}$ \\
\hline Neuropathy (n, \%) & $11(55.0)$ & $3(15.0)$ & $6(30.0)$ & NA \\
\hline Use of Antihypertensive (n, \%) & $31(38.8)$ & $21(26.2)$ & $28(35.0)^{\star \star}$ & $<0.001^{\star *}$ \\
\hline Use of Statin (n, \%) & $12(30.0)$ & $12(30.0)$ & $16(40.0)^{\star *}$ & $0.001^{* *}$ \\
\hline Use of AAS (n, \%) & $5(23.8)$ & $4(19.0)$ & $12(57.1)^{\star \star}$ & $0.001^{* *}$ \\
\hline Fasting glucose (mg/dL) & $176 \pm 76$ & $148 \pm 69^{*}$ & $149 \pm 68$ & $<0.001^{*}$ \\
\hline HbA1c (\%) & $8.1 \pm 1.3$ & $8.2 \pm 1.2$ & $9.8 \pm 2.2^{\star *}$ & $0.009^{\star \star}$ \\
\hline Serum creatinine (mg/dL) & $0.79(0.66-0.89)$ & $0.91(0.68-1.10)$ & $1.53(1.03-2.16)^{\star \star \dagger}$ & $\begin{array}{l}<0.001^{\star \star} \\
<0.001^{\dagger}\end{array}$ \\
\hline Urea (mg/dL) & $30 \pm 7$ & $35 \pm 9^{*}$ & $48 \pm 15^{\star \star+}$ & $\begin{aligned} & 0.001^{\star} \\
< & 0.001^{\star *} \\
< & 0.001^{\dagger}\end{aligned}$ \\
\hline Serum albumin (g/dL) & $4.1 \pm 0.4$ & $3.9 \pm 0.4$ & $3.9 \pm 0.4^{\star \star}$ & $0.007^{\star \star}$ \\
\hline Serum cystatin C (ng/mL) & $734(651-842)$ & $831(672-941)$ & $\begin{array}{c}1834 \\
(1074-2558)^{\star \star \dagger}\end{array}$ & $\begin{array}{l}<0.001^{\star \star} \\
<0.001^{\dagger}\end{array}$ \\
\hline eGFR-aMDRD (mL/min/1.73 m²) & $101(89-112)$ & $85(57-105)^{*}$ & $45(26-72)^{\star \star \dagger}$ & $\begin{aligned} & 0.004^{*} \\
<0.001^{\star *} & <0.001^{\dagger}\end{aligned}$ \\
\hline eGFR-CKD-EPIcr (mL/min/1.73 m²) & $114(104-123)$ & $98(74-118)^{*}$ & $49(28-83)^{\star \star \dagger}$ & $\begin{aligned} & 0.001^{\star} \\
&<0.001^{\star \star} \\
&<0.001^{\dagger}\end{aligned}$ \\
\hline eGFR-Tan (mL/min/1.73 m²) & $109(95-119)$ & $90(71-105)^{\star}$ & $41(27-74)^{\star \star \dagger}$ & $\begin{aligned} & 0.003^{*} \\
< & 0.001^{\star *} \\
< & 0.001^{\dagger}\end{aligned}$ \\
\hline $\begin{array}{l}\text { eGFR-Maclsaac } \\
\left(\mathrm{mL} / \mathrm{min} / 1.73 \mathrm{~m}^{2}\right)\end{array}$ & $112(97-121)$ & $92(73-107)^{*}$ & $43(30-77)^{\star \star \dagger}$ & $\begin{aligned} & 0.003^{*} \\
&< 0.001^{\star *} \\
&<0.001^{\dagger}\end{aligned}$ \\
\hline eGFR-CKD-EPIcys (mL/min/1.73 m²) & $112(89-129)$ & $86(67-106)^{\star}$ & $37(25-72)^{\star \star}$ & $\begin{aligned} & 0.002^{\star} \\
< & 0.001^{\star \star} \\
< & 0.001^{\dagger}\end{aligned}$ \\
\hline eGFR-CKD-EPIcrcys (mL/min/1.73 m²) & $112(97-125)$ & $88(68-116)^{\star}$ & $41(27-79)^{\star \star \dagger}$ & $\begin{aligned} & 0.006^{\star} \\
< & 0.001^{\star \star} \\
< & 0.001^{\dagger}\end{aligned}$ \\
\hline UAE (mg/g de creatinine) & $7(4-14)$ & $68(49-150)^{*}$ & $\begin{array}{c}926 \\
(475-1535)^{\star \star \dagger}\end{array}$ & $\begin{array}{l}<0.001^{*} \\
<0.001^{\star \star} \\
<0.001^{\dagger}\end{array}$ \\
\hline
\end{tabular}

Normally-distributed data were expressed as mean \pm SD and compared by ANOVA and T test. Not normally distributed data were expressed as median (percentiles $25 \%$ - $75 \%$ ) and compared by the Kruskal-Wallis $\mathrm{H}$ test and Mann-Whitney $\mathrm{U}$ test, followed by Bonferroni correction. Categorical variables were expressed as frequencies $\mathrm{n}(\%)$ and compared using the chi-square test $\left(\chi^{2}\right)$.

${ }^{*} \mathrm{P}<0.05$ for patients with microalbuminuria compared to patients with normoalbuminuria.

${ }^{* \star} P<0.05$ for patients with macroalbuminuria compared to patients with normoalbuminuria.

${ }^{\dagger} \mathrm{P}<0.05$ for patients with macroalbuminuria compared to patients with microalbuminuria. 
dex and Spearman correlation were used to assess the quality of the patients' classification. The classification of patients according to GFR estimated by aMDRD equation had a regular correlation with the classification of patients according UAE $(0.20 \leq \mathrm{k} \leq 0.39)$, while GFR estimated by CKD-EPIcr, CKD-EPIcrcys and cystatin C-based equations presented a moderate correlation $(0.40 \leq \mathrm{k} \leq 0.59)$. Spearman correlation has shown that GFR estimated by cystatin C-based equations presented a better correlation with UAE than GFR estimated by CKD-EPIcrcys and creatinine-based equations.

Patients were also classified into two groups according to GFR estimated by the formulas (eGFR $\geq 60$ and $<60 \mathrm{~mL} / \mathrm{min} / 1.73 \mathrm{~m}^{2}$ ) and GFR was associated to presence or absence of macroalbuminuria (Table 3 ). The reduced GFR estimated by all the formulas presented a significant association with macroalbuminuria, as observed by odds ratio analysis [OR of 14.8 $(5.7-38.9), 21.2(7.1-62.8), 17.9(6.3-51.0)$ and 31.9 (10.5 - 97.3) for aMDRD, CKD-EPIcr, CKDEPIcrcys and cystatin C-based equations, respectively].
It was also observed that the GFR estimated by cystatin C-based equations presented a better association with macroalbuminuria than the GFR estimated by CKDEPIcrcys and creatinine-based equations.

ROC curves were constructed to compare AUCs of GFR estimated by the formulas in reference to presence of macroalbuminuria and very similar AUCs were observed (Figure 1). However, it was verified that cystatin C-based equations presented higher AUCs than CKDEPIcrcys and creatinine-based equations. We also calculated sensitivity, specificity and accuracy for a cut-off < $60 \mathrm{~mL} / \mathrm{min} / 1.73 \mathrm{~m}^{2}$ and observed that aMDRD and cystatin C-based equations presented the same sensitivity to detect patients with macroalbuminuria, while CKDEPIcr and cystatin C-based equations presented the same specificity. However, aMDRD and CKD-EPIcrcys formulas presented a worse specificity than cystatin Cbased equations, while CKD-EPIcr and CKD-EPIcrcys formulas presented a worse sensitivity. Accuracy was very similar among the formulas, however, cystatin C-based equations presented higher accuracy than CKD-EPIcrcys and creatinine-based equations (Table 4).

Table 3. Comparison among GFR estimated by creatinine-based and cystatin C-based formulas according to albuminuria

\begin{tabular}{lcccc}
\hline Classification of patients & $\begin{array}{c}\text { Odds Ratio } \\
\text { (95\% Confidence Interval) }\end{array}$ & p & Kappa index & Spearman correlation \\
\hline Total $(\mathrm{n})$ & $14.8(5.7-38.9)$ & $<0.001$ & 0.326 & -0.508 \\
eGFR-AMDRD & $21.2(7.1-62.8)$ & $<0.001$ & 0.450 & -0.529 \\
eGFR-CKD-EPIcr & $31.9(10.5-97.3)$ & $<0.001$ & 0.433 & -0.609 \\
eGFR-Tan & $31.9(10.5-97.3)$ & $<0.001$ & 0.429 & -0.608 \\
eGFR-Maclsaac & $31.9(10.5-97.3)$ & $<0.001$ & 0.428 & -0.575 \\
eGFR-CKD-EPIcys & $17.9(6.3-51.0)$ & $<0.001$ & 0.422 & -0.558 \\
eGFR-CKD-EPIcrcys & & & & \\
\hline
\end{tabular}

GFR $<60 \mathrm{~mL} / \mathrm{min} / 1.73 \mathrm{~m}^{2}$ estimated by the formulas were compared to macroalbuminuria using the chi-square test $\left(\chi^{2}\right)$ and odds ratio (95\% confidence interval) was calculated. Classification of patients according to level of GFR decline ( $\geq 90 \mathrm{~mL} / \mathrm{min} / 1.73 \mathrm{~m}^{2} ; \geq 60$ and $\left.<90 \mathrm{~mL} / \mathrm{min} / 1.73 \mathrm{~m}^{2}<60 \mathrm{~mL} / \mathrm{min} / 1.73 \mathrm{~m}^{2}\right)$ were compared to classification of patients according to albuminuria (normalbuminuria, microalbuminuria and macroalbuminuria) using the chi-square test $\left(\chi^{2}\right)$ and Kappa index was calculated. Spearman correlation was calculated to compare non-categorized GFR estimated by formulas with non-categorized albuminuria.

Table 4. AUC of the GFR estimated by the formulas in reference to macroalbuminuria, sensitivity, specificity and accuracy for GFR $<60 \mathrm{~mL} / \mathrm{min} / 1.73 \mathrm{~m}{ }^{2}$

\begin{tabular}{lcccc}
\hline Formula & AUC & Sensitivity (\%) & Specificity (\%) & Accuracy (\%) \\
\hline eGFR-AMDRD & $0.832(0.726-0.937)$ & 68.8 & 87.1 & 82.4 \\
eGFR-CKD-EPIcr & $0.838(0.734-0.942)$ & 59.4 & 93.5 & 84.8 \\
eGFR-Tan & $0.894(0.827-0.960)$ & 68.8 & 93.5 & 87.2 \\
eGFR-Maclsaac & $0.894(0.827-0.960)$ & 68.8 & 93.5 & 87.2 \\
eGFR-CKD-EPIcys & $0.886(0.819-0.953)$ & 68.8 & 93.5 & 87.2 \\
eGFR-CKD-EPIcrcys & $0.840(0.739-0.940)$ & 59.4 & 92.5 & 84.0 \\
\hline
\end{tabular}

Area under curves (AUCs) are presented as median (95\% confidence interval). Sensitivity was defined as the percentage of patients with eGFR $<60 \mathrm{~mL} / \mathrm{min} / 1.73 \mathrm{~m}{ }^{2}$ among patients with macroalbuminuria. Specificity was defined as the percentage of patients with eGFR $\geq 60 \mathrm{~mL} / \mathrm{min} / 1.73 \mathrm{~m}^{2}$ among patients with normoalbuminuria or microalbuminuria. Accuracy was defined as the percentage of patients with eGFR $<60 \mathrm{~mL} / \mathrm{min} / 1.73 \mathrm{~m}^{2}$ and macroalbuminuria and with eGFR $\geq 60 \mathrm{~mL} / \mathrm{min} / 1.73 \mathrm{~m}^{2}$ and normoalbuminuria or microalbuminuria among all patients. 

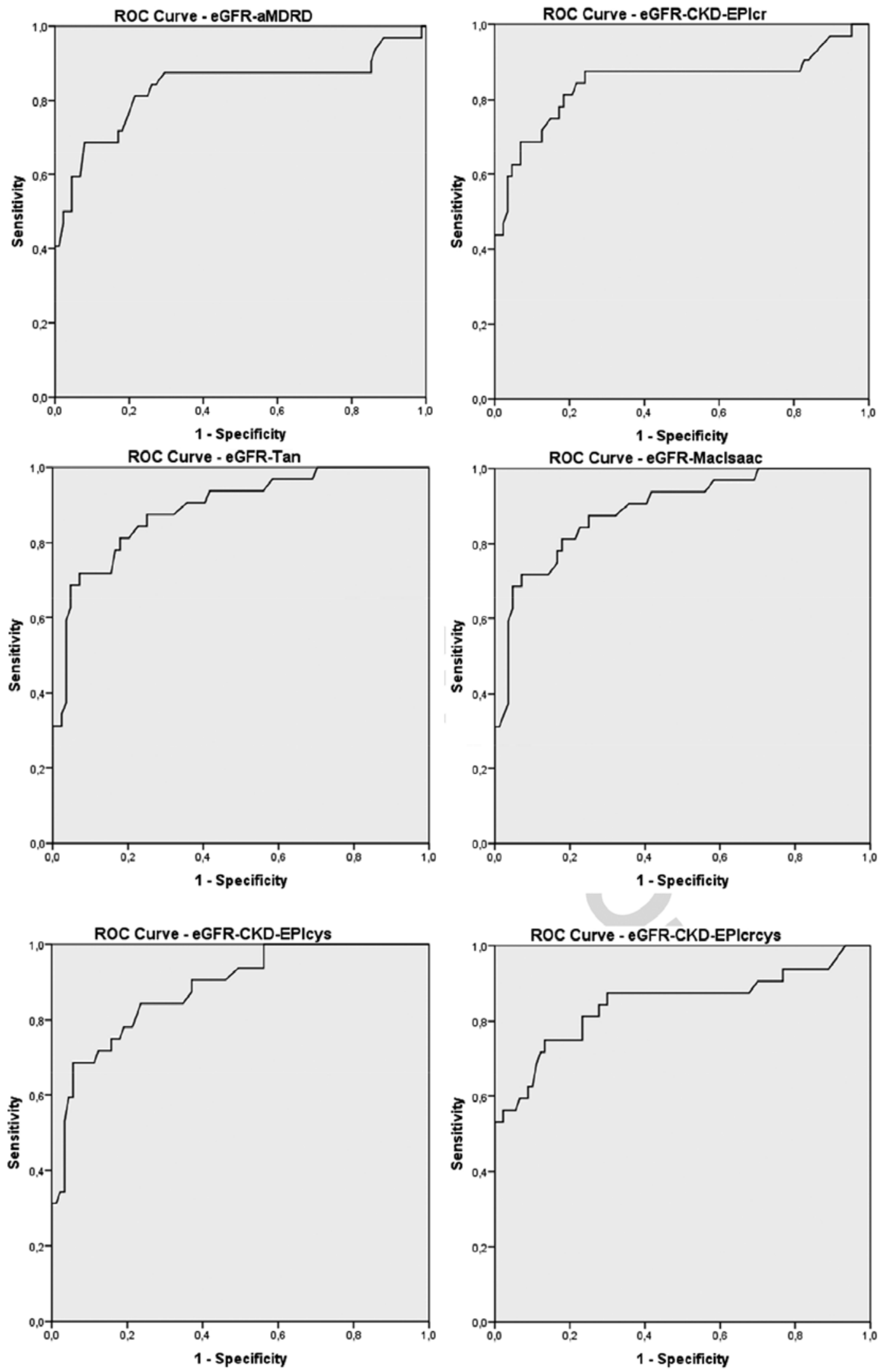

Figure 1. ROC curves of GFR estimated by creatinine-based and cystatin C-based formulas in reference to macroalbuminuria. 


\section{DISCUSSION}

Nephropathy is a very common complication in TIDM patients. Early detection of this complication is a relevant issue, since the majority of patients with nephropathy have hypertension, dyslipidemia and an increased risk of cardiovascular disease and renal failure (4). Several biomarkers and formulas based in these biomarkers may be used to estimate GFR and to evaluate renal function. However, all of them have limitations and there is still no ideal biomarker. Moreover, very few studies have compared their performance in TIDM patients. Here, we have compared two important biomarkers, creatinine and cystatin $\mathrm{C}$, and their most used derived formulas to estimate GFR. Furthermore, we evaluated the correlation of these formulas with the levels of UAE, a recognized biomarker of renal injury, in TIDM patients.

As expected, clinical findings differed in diabetic patients according to UAE. Increased frequencies of use of antihypertensive, statin and AAS were observed in patients with macroalbuminuria, as expected $(4,26)$. An increased frequency of retinopathy was also detected, which can be explained by the fact that retinopathy and nephropathy are both microvascular complications of diabetes and may have similar pathological mechanisms (26). The frequency of neuropathy was not associated with UAE. Since neuropathy is an early complication of diabetes, neuropatic involvement usually takes place before renal commitment. Therefore, neuropathy is usually present in both patients with and without nephropathy (24). Patients with macroalbuminuria also presented increased levels of HbAlc, indicating a poor glycemic control, which is indeed a risk factor for diabetic nephropathy, and decreased BMI, since malnutrition is common conditions in patients with renal disease $(4,26)$.

In this study, patients with normoalbuminuria, microalbuminuria and macroalbuminuria could be properly distinguished, regardless the creatinine-based equation used to estimate GFR, although serum creatinine was not discriminatory enough to differentiate patients with normoalbuminuria and microalbuminuria. This result is due to the fact that serum creatinine is affected by several other factors besides GFR and it is less sensitive to detect mild renal dysfunction. It has been demonstrated that GFR declines to approximately half the normal level before serum creatinine rises above the upper limit of normality (27). GFR as esti- mated by all cystatin C-based equations also differed according to the levels of UAE, while plasma cystatin C did not distinguish patients with normoalbuminuria and microalbuminuria in this study. It has been demonstrated that cystatin $\mathrm{C}$ is a better biomarker to detect early renal dysfunction in diabetic patients with normoalbuminuria (21). Therefore, its levels could already be elevated in patients with normoalbuminuria that present mild decline of GFR. In agreement, some studies have shown that serial measurements of plasma cystatin C can be useful to detect early renal dysfunction in TIDM patients $(28,29)$.

Some patients with normoalbuminuria presented reduced GFR, independent of the equation used to estimate GFR. Decline in GFR has been detected in TIDM and DM2 patients with normoalbuminuria $(30,31)$. While this finding may suggest the presence of renal dysfunction in the absence of albuminuria, the decline of GFR might also be due to underestimation of GFR by the equations used. We have observed that when aMDRD equation was used to estimate GFR, an increased number of patients with normoalbuminuria presented reduced GFR. This is in agreement with other studies, which have demonstrated that this formula tends to underestimate GFR in patients with normal renal function $(9,29)$.

Regardless of the equation used to estimate GFR, some patients with macroalbuminuria presented normal GFR or just a mild decline in GFR. This result suggests that simultaneous assessment of UAE and GFR are complementary for diagnosis of TIDM patients with nephropathy. Many patients with microalbuminuria also presented normal GFR, regardless of the equation used to estimate GFR, which can be explained by the fact that microalbuminuria is an earlier biomarker of renal injury and a risk factor for progression of nephropathy $(6,32)$.

The classification of patients according to GFR as estimated by aMDRD equation presented the worst correlation with the UAE classification of patients, while CKD-EPIcr formula presented the best correlation, according to Kappa index evaluation. Among creatinine-based equations, aMDRD formula also presented a worse accuracy to detect patients with macroalbuminuria, as compared to CKD-EPIcr formula. Besides, CKD-EPIcr formula has shown better association with UAE than aMDRD equation, by applying Spearman Correlation. This result is in agreement with other studies, which have demonstrated that CKD-EPIcr 
equation is more accurate than aMDRD formula to evaluate renal function (12,33-35). Indeed, National Kidney Foundation has recently recommended the use of CKD-EPIcr formula to evaluate GFR (35).

The odds ratio analysis has demonstrated that the GFR $<60 \mathrm{~mL} / \mathrm{min} / 1.73 \mathrm{~m}^{2}$ estimated by cystatin Cbased and creatinine-based equations presented similar associations with macroalbuminuria, indicating that a reduced GFR estimated by any of these formulas is significantly associated with renal injury. However, cystatin C-based equations presented a slightly increased association with macroalbuminuria than CKD-EPIcrcys and creatinine-based equations. Spearman Correlation and ROC curves comparing AUCs for creatinine-based and cystatin C-based formulas were also very similar to each other. However, cystatin C-based equations presented a slightly better correlation with UAE and higher AUCs to detect patients with macroalbuminuria than $\mathrm{CKD}$ EPIcrcys and creatinine-based equations. Although cystatin C-based equations have presented the same sensitivity of aMDRD formula and the same specificity of CKD-EPIcr formula to detect patients with macroalbuminuria, they have showed a better specificity than aMDRD and CKD-EPIcrcys formulas, a better sensitivity than CKD-EPIcr and CKD-EPIcrcys formulas and a higher accuracy than these formulas. These data suggest a better association between cystatin C-based formulas with the presence of renal injury. In agreement, Yoo and cols. (36) have demonstrated that the annual change in GFR estimated by cystatin C-based formula reflected the progression of albuminuria more accurately than GFR estimated by aMDRD formula in DM2 patients.

Nonetheless, the superiority of cystatin C-based equations to evaluate the presence of renal dysfunction in diabetic patients is still controversial. Thus, some authors have suggested that cystatin C-based formulas and serum cystatin $\mathrm{C}$ improve the assessment of renal function of these patients compared to creatinine-based formulas $(18,28,29,37,38)$ while others suggest that they have the same efficacy to evaluate renal function of diabetic patients $(39,40)$. Therefore, further studies are still necessary to elucidate this issue and to assess whether cystatin C-based equations really improve the assessment of GFR or not. Furthermore, it is extremely important to evaluate the performance of creatininebased and cystatin C-based formulas to estimate GFR in patients with different stages of UAE, since the evaluation of GFR and UAE are complementary to early detection of renal disease.
The transversal design of this study is a limitation, since longitudinal determinations of the GFR are more relevant for monitoring renal function than a unique measure. Indeed, it has been demonstrated that serial measurements of cystatin $\mathrm{C}$ may be more useful for evaluation of early renal function decline than GFR estimated by creatinine-based equations (29). Nevertheless, further longitudinal studies with larger sample size may be still necessary. The lack of a gold standard biomarker of GFR, such as inulin clearance, may be raised as another limitation of this study. However, measurement of inulin clearance requires patient hospitalization, which delays the diagnosis and reduces their feasibility in the routine management of patients with renal dysfunction. Given to their simplicity and accessibility, evaluation of renal function is more frequently performed through albuminuria and creatinine-based and cystatin C-based formulas. Therefore, comparisons of creatinine-based and cystatin C-based formulas with albuminuria are still relevant, helping clinicians to evaluate their utility and limitations for early detection of renal injury in TIDM patients.

In agreement, our results suggest that creatininebased and cystatin C-based equations were equally able to estimate GFR in TIDM patients correlated with different levels of UAE. However, cystatin C-based equations presented best accuracy to detect macroalbuminuria in TIDM patients. Although cystatin $\mathrm{C}$ is not always available in clinical practice, cystatin C-based equations should be considered in the clinical routine in order to increase the possibility of early diagnostic of chronic renal disease. We emphasize that further studies aiming to discover new renal biomarkers are extremely important, since there is still no ideal biomarker to evaluate GFR and to detect early decline of renal function.

Acknowlegments: the authors thank Fundação de Amparo à Pesquisa do Estado de Minas Gerais (Fapemig), Coordenação de Aperfeiçoamento de Pessoal de Nível Superior (Capes) and Conselho Nacional de Desenvolvimento Científico e Tecnológico $(\mathrm{CNPq}) /$ Brazil.

Disclosure: no potential conflict of interest relevant to this article was reported.

\section{REFERENCES}

1. Marshall SM. Recent advances in diabetic nephropathy. Postgrad Med J. 2004;80:624-33.

2. Karnib HH, Ziyadeh FN. The cardiorenal syndrome in diabetes mellitus. Diabetes Res Clin Pract. 2010;89:201-8. 
3. Yamagishi S, Matsui T. Advanced glycation end products, oxidative stress and diabetic nephropathy. Oxid Med Cell Longev. 2010;3:101-8.

4. Gross JL, de Azevedo MJ, Silveiro SP, Canani LH, Caramori ML, Zelmanovitz T. Diabetic nephropathy: diagnosis, prevention, and treatment. Diabetes Care. 2005;28:164-76.

5. Schernthaner G. Kidney disease in diabetology: lessons from 2008. Nephrol Dial Transplant. 2009; 24:396-9.

6. Reutens AT. Epidemiology of diabetic kidney disease. Med Clin North Am. 2013; 97:1-18.

7. Levey A, Greene T, Kusek J, Beck G, Group M. A simplified equation to predict glomerular filtration rate from serum creatinine [Abstract]. J Am Soc Nephrol. 2000;11:A0828.

8. Cockcroft DW, Gault MH. Prediction of creatinine clearance from serum creatinine. Nephron. 1976;16:31-41.

9. Maclsaac RJ, Premaratne E, Jerums G. Estimating glomerular filtration rate in diabetes using serum cystatin C. Clin Biochem Rev. 2011;32:61-7.

10. Cirillo M. Evaluation of glomerular filtration rate and of albuminuria/proteinuria. J Nephrol. 2010;23:125-32.

11. Massey D. Commentary: clinical diagnostic use of cystatin C. J Clin Lab Anal. 2004;18:55-60.

12. National Disease: Improving Global Outcomes (KDIGO) CKD Work Group. KDIGO clinical practive guideline for the evaluation and management of chronic kidney disease. Kidney Int Suppl. 2013;3:1-150.

13. Levey AS, Stevens LA, Schmid CH, Zhang YL, Castro AF 3rd, Feldman HI, et al.; CKD-EPI (Chronic Kidney Disease Epidemiology Collaboration). A new equation to estimate glomerular filtration rate. Ann Intern Med. 2009;150:604-12.

14. Eriksen BO, Mathisen UD, Melsom T, Ingebretsen OC, JenssenTG, Njølstad I, et al. The role of cystatin C in improving GFR estimation in the general population. Am J Kidney Dis. 2012;59:32-40.

15. Levey AS, Inker LA, Coresh J. GFR estimation: from physiology to public health. Am J Kidney Dis. 2014;63:820-34.

16. Stevens LA, Coresh J, Schmid CH, Feldman HI, Froissart M, Kusek $\mathrm{J}$, et al. Estimating GFR using serum cystatin $\mathrm{C}$ alone and in combination with serum creatinine: a pooled analysis of 3,418 individuals with CKD. Am J Kidney Dis. 2008;51:395-406.

17. Macisaac RJ,Tsalamandris C, Thomas MC, Premaratne E, Panagiotopoulos S, Smith TJ, et al. Estimating glomerular filtration rate in diabetes: a comparison of cystatin-C- and creatinine-based methods. Diabetologia. 2006;49:1686-9.

18. Tan GD, Lewis AV, James TJ, Altmann P, Taylor RP, Levy JC. Clinical usefulness of cystatin $C$ for the estimation of glomerular filtration rate in type 1 diabetes: reproducibility and accuracy compared with standard measures and iohexol clearance. Diabetes Care. 2002;25:2004-9.

19. Woo KS, Choi JL, Kim BR, Kim JE, Han JY. Clinical usefulness of serum cystatin $\mathrm{C}$ as a marker of renal function. Diabetes Metab J. 2014;38:278-84.

20. Cabarkapa V. Cystatin $\mathrm{C}$ - more than the marker of the glomerular filtration rate. Med Pregl. 2015; 68:173-9.

21. Jeon YK, Kim MR, Huh JE, Mok JY, Song SH, Kim SS, et al. Cystatin $\mathrm{C}$ as an early biomarker of nephropathy in patients with type 2 diabetes. J Korean Med Sci. 2011;26:258-63.

22. Nitta K, Hayashi T, Uchida K, Honda K, Tsukada M, Sekine S, et al. Serum cystatin $\mathrm{C}$ concentration as a marker of glomerular filtration rate in patients with various renal diseases. Intern Med. 2002;41:931-5.

23. Kazama JJ, Kutsuwada K, Ataka K, Maruyama H, Gejyo F. Serum cystatin $C$ reliably detects renal dysfunction in patients with various renal diseases. Nephron. 2002;91:13-20.
24. American Diabetes Association. Diagnosis and classification of diabetes mellitus. Diabetes Care. 2013;36:dc13-S067.

25. Levey AS, Coresh J, Greene T, Marsh J, Stevens LA, Kusek JW, et al. Expressing the Modification of Diet in Renal Disease Study equation for estimating glomerular filtration rate with standardized serum creatinine values. Clin Chem. 2007;53:766-72.

26. Raile K, Galler A, Hofer S, Herbst A, Dunstheimer D, Busch P, et al. Diabetic nephropathy in 27,805 children, adolescents, and adults with type 1 diabetes: effect of diabetes duration, A1C, hypertension, dyslipidemia, diabetes onset, and sex. Diabetes Care. 2007;30:2523-8.

27. Levey AS, Coresh J, Balk E, Kausz AT, Levin A, Steffes MW, et al. National Kidney Foundation practice guidelines for chronic kidney disease: evaluation, classification, and stratification. Ann Intern Med. 2003;139:137-47.

28. Perkins BA, Krolewski AS. Early nephropathy in type 1 diabetes: the importance of early renal function decline. Curr Opin Nephrol Hypertens. 2009;18:233-40.

29. Premaratne E, Maclsaac RJ, Finch S, Panagiotopoulos S, Ekinci E, Jerums $\mathrm{G}$. Serial measurements of cystatins $\mathrm{C}$ are more accurate than creatinine-based methods in detecting declining renal function in type 1 diabetes. Diabetes Care. 2008;31:971-3.

30. Caramori ML, Fioretto $\mathrm{P}$, Mauer M. Low glomerular filtration rate in normoalbuminuric type 1 diabetic patients: an indicator of more advanced glomerular lesions. Diabetes. 2003;52:1036-40.

31. Kramer HJ, Nguyen QD, Curhan G, Hsu CY. Renal insufficiency in the absence of albuminuria and retinopathy among adults with type 2 diabetes mellitus. JAMA. 2003;289:3273-7.

32. Maclsaac RJ, Ekinci El, Jerums G. Markers of and risk factors for the development and progression of diabetic kidney disease. Am J Kidney Dis. 2014;63:S39-62.

33. Murata K, Baumann NA, Saenger AK, Larson TS, Rule AD, Lieske JC. Relative performance of the MDRD and CKD-EPI equations for estimating glomerular filtration rate among patients with varied clinical presentations. Clin J Am Soc Nephrol. 2011;6:1963-72

34. Cirillo M, Lombardi C, Luciano MG, Bilancio G, Anastasio P, De Santo NG. Estimation of GFR: a comparision of new and establiched equations. Am J Kidney Dis. 2010;56:802-4.

35. Vucic Lovrencic M, Radisic Biljak V, Bozicevic S, Prasek M, Pavkovic $P$, Knotek M. Estimating glomerular filtration rate (GFR) in diabetes: the performance of MDRD and CKD-EPI equations in patients with various degrees of albuminuria. Clin Biochem. 2012;45:1694-6.

36. Yoo JS, LeeYM, Lee EH, Kim JW, Lee SY, Jeong KC, et al. Serum cystatin $\mathrm{C}$ reflects the progress of albuminuria. Diabetes Metab J. 2011;35:602-9.

37. Pucci L, Triscornia S, Lucchesi D, Fotino C, Pellegrini G, Pardini E. Cystatin $C$ and estimates of renal function: searching for a better measure of kidney function in diabetic patients. Clin Chem. 2007;53:480-8.

38. Krolewski AS, Warram JH, Forsblom C, Smiles AM, Thorn L, Skupien J, et al. Serum concentration of cystatin $\mathrm{C}$ and risk of endstage renal disease in diabetes. Diabetes Care. 2012;35:2311-6.

39. Li HX, Xu GB, Wang XJ, Zhang XC, Yang JM. Diagnostic accuracy of various glomerular filtration rates estimating equations in patients with chronic kidney disease and diabetes. Chin Med J. 2010;123:745-51.

40. Iliadis F, Didangelos T, Ntemka A, Makedou A, Moralidis E, Gotzamani-Psarakou $A$, et al. Glomerular filtration rate estimation in patients with type 2 diabetes: creatinine- or cystatin C-based equations? Diabetologia. 2011;54:2987-94. 